\title{
Support to scale antibiotic stewardship in long-term care homes: how much is enough?
}

\author{
Julia Szymczak (D) , ${ }^{1}$ Barbara Trautner (D) ${ }^{2,3}$
}

'Biostatistics, Epidemiology and Informatics, University of Pennsylvania Perelman School of Medicine, Philadelphia Pennsylvania, USA

${ }^{2}$ Center for Innovations in Quality, Effectiveness, and Safety, Michael E. DeBakey VA Medical Center, Houston, Texas, USA

${ }^{3}$ Section of Health Services Research, Departments of Medicine and Surgery, Baylor College of Medicine, Houston, Texas, USA

\section{Correspondence to}

Dr Julia Szymczak, Biostatistics, Epidemiology and Informatics, University of Pennsylvania Perelman School of Medicine, Philadelphia, PA 19104, USA; jszymcza@pennmedicine.upenn. edu

Accepted 25 May 2021

\section{SLinked}

http://dx.doi.org/10.1136/ bmjqs-2020-012226

\section{Check for updates}

(c) Author(s) (or their employer(s)) 2021. No commercial re-use. See rights and permissions. Published by BMJ.

To cite: Szymczak J,

Trautner B. BMJ Qual Saf Epub ahead of print: [please include Day Month Year].

doi:10.1136/

bmjgs-2021-013073
As evidence demonstrating the positive impact of antibiotic stewardship interventions grows, there is an urgent need to understand how these efforts can be replicated in other settings ('spread') and how infrastructure can be developed to support broader implementation across large systems of care ('scale'). ${ }^{12}$ In addition to ensuring that individual patients are protected from adverse effects of unnecessary antibiotics, there is a societal imperative to spread and scale stewardship such that it reaches large numbers of people, as misuse of antibiotics has a ripple effect across populations through the emergence of resistant infections. Identifying strategies to move stewardship beyond the controlled, well-resourced world of research to the real world will ensure that the benefit of investment in research is maximised while population harms from antibiotic overuse are minimised. ${ }^{3}$

In this issue of BMJ Quality and Safety, Chambers and colleagues report the results of a controlled before-and-after study, accompanied by a process evaluation, assessing the impact of virtual learning collaboratives to scale an antibiotic stewardship programme that had previously been demonstrated to be effective in long-term care homes (LTCHs) across Ontario. ${ }^{4}$ Public Health Ontario (PHO), an arm's length governmental body that provides scientific expertise to support healthcare in the province, previously developed the Urinary Tract Infection (UTI) Program to reduce inappropriate urine culturing and unnecessary antibiotic prescribing for asymptomatic bacteriuria in non-catheterised residents of LTCHs. The multimodal UTI Program consists of written guidance for LTCHs about how to adopt best practices through a list of specific implementation strategies (eg, readiness strategies, education, monitoring) and a suite of tools to support these efforts (eg, fact sheets, posters, communication material for patients and families, process surveillance forms, assessment algorithm for UTIs). ${ }^{5}$ After demonstrating effectiveness at reducing urine culturing and antibiotic use in a small pilot of 10 facilities, ${ }^{6} \mathrm{PHO}$ sought to implement the programme more widely. They selected virtual learning collaboratives as a strategy to scale the UTI Program to all LTCHs in Ontario.

Learning collaboratives are a commonly used implementation and quality improvement strategy. Teams from multiple organisations engage in repeated episodes of shared learning, group discussion, skill building and data sharing under the guidance of expert faculty, typically conducted face to face. ${ }^{7}$ Virtual alternatives have been proposed, to reduce the cost of participation and increase the speed of translating evidence into practice, although there is limited evidence of their effectiveness. ${ }^{8}$ Known barriers to virtual collaboratives include lack of engagement and accountability, time constraints and scheduling, personnel turnover, lack of clarity about expectations and difficulty navigating technology. ${ }^{9} 10$ However, virtual collaboratives are an appealing strategy for scale of stewardship interventions in LTCHs because they minimise stakeholder time away from the clinical setting (an important consideration for contexts with frequent staffing shortages), allow remote facilities efficient access to collaborative activities and facilitate ongoing improvement work when physical distancing measures prohibit gathering (such as in the current pandemic).

While appealing in theory, how well do virtual collaboratives work to support scale and improvement in long-term care 


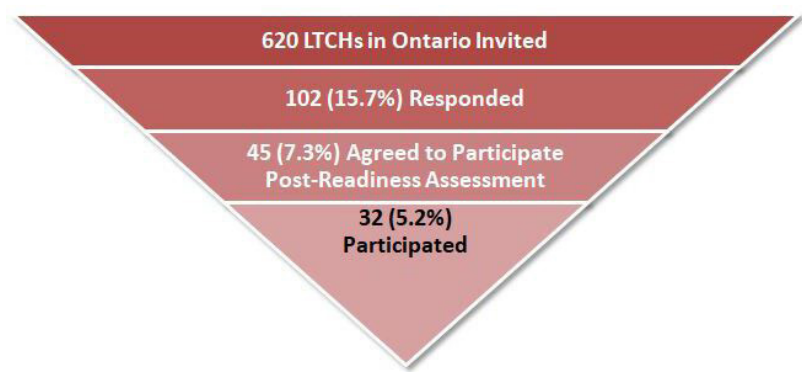

Figure 1 Attrition of long-term care home (LTCH) engagement in scale of the Urinary Tract Infection Program over time. ${ }^{4}$

settings? These can be particularly challenging places to implement antibiotic stewardship interventions due to financial constraints, frequent staff turnover and family pressure to prescribe. ${ }^{11}$ LTCHs also often lack access to physicians or pharmacists with antibiotic stewardship expertise, capacity to track and report antibiotic use data and on-site diagnostic laboratory services. ${ }^{1213}$ Variable uptake and low engagement with stewardship interventions by LTCHs are common even in the relatively well-resourced setting of research. ${ }^{14}$

Against this backdrop, the findings of positive change in key outcome metrics from the 32 of 620 long-term care facilities in Ontario that engaged with the UTI Program via virtual learning collaboratives, as described by Chambers and colleagues, inspire a feeling of measured hope. LTCHs that participated in the virtual learning collaboratives had significantly greater decreases in rates of urine culturing and urinary antibiotic prescriptions per 1000 resident days, compared with matched controls. The magnitude of change observed was modest (a difference of 1 urine culture per 1000 resident days, and 0.5 day of urinary antibiotic use per 1000 resident days) but a difference-indifference analysis demonstrated a significant benefit of participation in the virtual learning collaboratives. The change in the rate of urine cultures performed was $19 \%$ lower while urinary antibiotic prescriptions were $13 \%$ lower in those LTCHs that participated than in the control group $(\mathrm{p}<0.0001)$.

Interestingly, the accompanying process evaluation suggests that the benefit of participation in the virtual learning collaboratives is not dependent on attendance at all sessions or complete adoption of all the recommended implementation strategies. The virtual learning collaboratives comprised three sessions. Only $36 \%$ of
LTCHs were represented at all sessions. Chambers and colleagues observed that there was no significant difference in impact between LTCHs that attended all sessions and those that attended only some. This raises questions about the 'dose' of collaborative participation needed to derive benefit, or whether facilities that fully engage in learning collaboratives on an ongoing basis have intrinsic organisational characteristics that predispose to success.

In this real-world intervention, LTCHs were given free choice about which implementation strategies to adopt. It is not surprising that there was variation observed in their use, given that LTCHs have different structures and needs. ${ }^{15} 16$ What is interesting is that the implementation strategies least commonly used in the study-readiness strategies to engage prescribers, and audit of performance-are traditionally considered to be the most impactful on changing antibiotic prescribing practices. ${ }^{14} 17$ We do not know why LTCHs selected the implementation strategies that they did, but we hypothesise that their choices had to do with familiarity ( $84 \%$ chose education) and level of personnel effort required (only $47 \%$ built a threeperson implementation team). What is reassuring is that these LTCHs found success through the use of strategies that were considered appropriate for their local context.

While this study demonstrates the effectiveness of virtual learning collaboratives as a technique to scale antibiotic stewardship in the real world, some key questions remain about how a greater degree of engagement with voluntary antibiotic stewardship interventions can be secured across a large number of facilities. Chambers and colleagues started by approaching all $620 \mathrm{LTCH}$ in Ontario. The engagement of LTCHs from recruitment to full participation involved considerable attrition over time, with only $5.2 \%$ of the LTCHs in Ontario ultimately engaging with the UTI Program via the virtual learning collaboratives (figure 1 ).

There is little information reported in the paper about why some LTCHs declined participation and none about why some withdrew. Truly scaling stewardship via broad implementation requires a greater understanding of how to get LTCHs to engage with free, locally adaptive, effective and minimally timeintensive programmes.

Table 1 Outer context domains to support scale in antibiotic stewardship in long-term care homes (LTCHs)

\begin{tabular}{ll}
\hline Incentives and policy & LTCH antibiotic stewardship honour roll with tiered system of status. ${ }^{12}$ \\
Performance metrics and data feedback & Regulatory requirement that LTCHs document antibiotic stewardship activities. \\
& Quarterly reports to LTCH on rates of urine culturing and antibiotic use. \\
Interorganisational networks & Regional dashboards with performance measures to promote peer pressure. \\
& address stewardship across the care continuum..$^{20}$ \\
& Collaboration with LTCH and their contracted laboratory to develop facility-specific antibiograms. ${ }^{12}$ \\
\hline
\end{tabular}


A consideration of the public health infrastructure in Ontario surrounding these virtual learning collaboratives provides important information about the features of complex interdependent systems of care that may support or impede scale in stewardship. ${ }^{18}$ PHO promotes scaling of public health interventions, with considerable reach across a network of LTCHs via existing regional infection prevention and control support teams situated across the province, expertise in stewardship, trained facilitators and access to comprehensive provincial administrative data to support the outcome assessment. There are other features of the outer context that, if present, may have also encouraged greater engagement by LTCHs in the virtual learning collaboratives (table 1). Encouraging busy, often under-resourced LTCHs to participate in stewardship could be bolstered by policies at the system level that incentivise engagement through regulatory requirements, peer pressure, reputational incentives, performance metrics and leveraging stewardship expertise through pre-existing interorganisational networks.

Spread and scale of interventions to change clinical practice is challenging in general, but especially complex for antibiotic stewardship in the LTCH setting. Changing antibiotic prescribing requires the engagement of multiple stakeholders with diverse priorities, modifying deeply ingrained clinician and patient behaviours, coordinating collective action across institutions within a region, accessing valid, informative and timely antibiotic use metrics, securing leadership accountability for performance and contending with resource limitations. Virtual learning collaboratives are one low-resource intensive technique that intermediary organisations, public health agencies, regulatory bodies and healthcare systems can use to spread best practices in antibiotic stewardship to the many sites of care in which patients could benefit. More research is needed to understand how to engage a larger number of organisations with these voluntary programmes such that the principles of stewardship can be embedded in all settings where antibiotics are used.

Twitter Julia Szymczak @JulieSzymczak and Barbara Trautner @bwtrautner

Funding JS receives funding from the US Centers for Disease Control and Prevention Cooperative Agreement FOA\#CK16004-Epicenters for the Prevention of Healthcare-Associated Infections. BT's work is supported in part by the Houston Veterans Affairs Health Services Research \& Development Center for Innovations in Quality, Effectiveness and Safety (CIN 13-413).

Disclaimer The views expressed in this article are those of the authors and do not necessarily reflect the position or policy of the Department of Veterans Affairs or the US government.

Competing interests None declared.

Patient consent for publication Not required.
Provenance and peer review Commissioned; internally peer reviewed.

\section{ORCID iDs}

Julia Szymczak http://orcid.org/0000-0002-3230-8670

Barbara Trautner http://orcid.org/0000-0001-7986-9099

\section{REFERENCES}

1 Morris AM, Calderwood MS, Fridkin SK, et al. Research needs in antibiotic stewardship. Infect Control Hosp Epidemiol 2019;40:1334-43.

2 Greenhalgh T, Papoutsi C. Spreading and scaling up innovation and improvement. BMJ;2019:12068.

3 Trautner BW, Naik AD. Taking a ride on the stewardship side of long-term care: the Cadillac and the Camry. JAMA Netw Open 2019;2:e199515.

4 Chambers A, Chen C, Brown KA, et al. Virtual learning collaboratives to improve urine culturing and antibiotic prescribing in long-term care: controlled before-and-after study. BMJ Qual Saf 2021:bmjqs-2020-012226.

5 Ontario PH. Urinary tract infection program, 2019. Available: https:/www.publichealthontario.ca/en/health-topics/ antimicrobial-stewardship/uti-program [Accessed $25 \mathrm{Apr}$ 2021].

6 Brown KA, Chambers A, MacFarlane S, et al. Reducing unnecessary urine culturing and antibiotic overprescribing in long-term care: a before-and-after analysis. CMAJ Open 2019; 7:E174-81.

7 Nadeem E, Olin SS, Hill LC, et al. Understanding the components of quality improvement Collaboratives: a systematic literature review. Milbank Q 2013;91:354-94.

8 Zubkoff L, Neily J, Mills PD. How to do a virtual breakthrough series collaborative. J Med Syst 2019;43:27.

9 Zubkoff L, Neily J, Quigley P, et al. Virtual breakthrough series, part 2: improving fall prevention practices in the Veterans health administration. Jt Comm J Qual Patient Saf 2016;42:497-AP12.

$10 \mathrm{Kamal} \mathrm{AH,} \mathrm{Bossley} \mathrm{H,} \mathrm{Blum} \mathrm{R,} \mathrm{et} \mathrm{al.} \mathrm{Evaluation} \mathrm{of} \mathrm{a} \mathrm{virtual}$ learning collaborative to integrate palliative care into routine oncology practice. JCO Oncol Pract 2020;16:e1371-7.

11 Katz MJ, Gurses AP, Tamma PD, et al. Implementing antimicrobial stewardship in long-term care settings: an integrative review using a human factors approach. Clin Infect Dis 2017;65:1943-51.

12 Adre C, Jump RLP, Spires SS. Recommendations for improving antimicrobial stewardship in long-term care settings through collaboration. Infect Dis Clin North Am 2020;34:129-43.

13 Davidson HE, Jump RLP. Challenges in tracking and reporting antibiotic use in long-term care. J Am Med Dir Assoc 2020;21:1191-6.

14 Raban MZ, Gasparini C, Li L, et al. Effectiveness of interventions targeting antibiotic use in long-term aged care facilities: a systematic review and meta-analysis. BMJ Open 2020;10:e028494.

15 Palms DL, Kabbani S, Bell JM, et al. Implementation of the core elements of antibiotic stewardship in nursing homes enrolled in the National healthcare safety network. Clin Infect Dis 2019;69:1235-8.

16 Wu JH-C, Langford BJ, Daneman N, et al. Antimicrobial stewardship programs in long-term care settings: a meta-analysis and systematic review. J Am Geriatr Soc 2019;67:392-9.

17 Goebel MC, Trautner BW, Wang Y, et al. Organizational readiness assessment in acute and long-term care has important 


\section{Editorial}

implications for antibiotic stewardship for asymptomatic bacteriuria. Am J Infect Control 2020;48:1322-8.

18 Greenhalgh T, Robert G, Macfarlane F, et al. Diffusion of innovations in service organizations: systematic review and recommendations. Milbank Q 2004;82:581-629.
19 Gross PA, Patel B. Reducing antibiotic overuse: a call for a national performance measure for not treating asymptomatic bacteriuria. Clin Infect Dis 2007;45:1335-7.

20 Crnich CJ, Malani P. Opportunities to improve antimicrobial use in US nursing homes. JAMA 2021;325:1259-60. 